Bull. Chem. Soc. Ethiop. 2017, 31(3), 519-534.

ISSN 1011-3924

(c) 2017 Chemical Society of Ethiopia and The Authors

Printed in Ethiopia

DOI: http://dx.doi.org/10.4314/bcse.v31i3.16

\title{
USES OF ACETOACETANILIDE FOR THE SYNTHESIS OF THIOPHENE DERIVATIVES WITH CYTOTOXIC ACTIVITIES
}

\author{
Rehab A. Ibrahim* \\ Higher Institute of Engineering and Technology, El-Tagammoe El-Khames, New Cairo, Egypt
}

(Received October 21, 2017; Revised December 13, 2017; Accepted December 16, 2017)

\begin{abstract}
The reaction of acetoacetanilide 1 with elemental sulfur and malononitrile 2 gave the thiophene derivative 3. The latter was the key starting material for the synthesis of different thiophene and fused derivatives through its reaction with aryldiazonium chloride, benzaldehyde and acetic anhydride. Moreover, the reaction with phenylisothiocyanate produced fused derivative. The reaction of compound $\mathbf{3}$ with elemental sulfur produced dithiophene derivative. In addition, the reaction of compound $\mathbf{3}$ with ethylcyanoacetate produced $\mathrm{N}$ cyanoacetamide derivative that was capable for further heterocyclizations through its reaction with different reagents. The cytotoxicity of the newly synthesized products was evaluated against the three cancer cell lines namely MCF-7 (breast adenocarcinoma), NCI-H460 (non-small cell lung cancer), and SF-268 (CNS cancer) where some compounds showed optimal cytotoxicity. The results showed that compounds $\mathbf{5 a}, \mathbf{5 b}, \mathbf{8}, \mathbf{2 2}$, and $\mathbf{2 3}$ exhibited optimal cytotoxic effect against cancer cell lines.
\end{abstract}

KEY WORDS: Thiophene, Thieno[2,3- $d]$ pyrimidine, Coumarin, Cytotoxicity

\section{INTRODUCTION}

Thiophene derivatives represent a class of important and well-studied heterocycles [1]. The interest in this kind of heterocycles has spread from early dye chemistry [2] to modern drug design [3], biodiagnostics [4], electronic and optoelectronic devices [5], conductivity-based sensors [6], and self-assembled superstructures [7]. The general synthetic approaches to such kind of compounds either involve the functionalization at the positions $a$ and $b$ to the sulfur atom of the pre-constructed thiophene nucleus [8], or the construction of thiophene ring from appropriately substituted open chain precursors [9]. The latter becomes much attractive for its general applicability to achieve more complicated substitution patterns [10]. Gewald and coworkers developed the synthesis of 2-aminothiophenes from the multicomponent condensation of ketones or aldehydes, cyanoacetate and elemental sulfur [11]. Later on, there are several reviews and papers reported on the variations and improvements on the originally published Gewald's synthesis of polysubstituted thiophenes [12]. Recently, we were involved through comprehensive program involving the synthesis of thiophene $[13,14]$ derivatives together with their further reactions with chemical reagents to give heterocyclic and fused heterocyclic derivatives with antitumor activities. Moreover, we synthesized different thiophene derivatives that have been screened for antitumor activity against breast adenocarcinoma (MCF-7), nonsmall cell lung cancer (NCI-H460) and CNS cancer (SF-268); this is besides studying their cytotoxicity against the normal human cell line normal fibroblast cells WI 38 [15]. In this work we make extension for this program through the synthesis of thiophene derivatives using acetoacetanilide and maononitrile in the presence of elemental sulfur to produce potentially antitumor thiophene derivatives [16-23].

*Corresponding author. E-mail: drrehab223@yahoo.com

This work is licensed under the Creative Commons Attribution 4.0 International License 


\section{RESULTS AND DISCUSSION}

In the present work, acetaacetanilide 1 reacted with elemental sulfur and malononitrile $\mathbf{2}$ in the presence of triethylamine to give the thiophene derivative $\mathbf{3}$. The structure of compound $\mathbf{3}$ was based on analytical and spectral data. Thus, the ${ }^{1} \mathrm{H}$ NMR spectrum showed one singlet at $\delta 2.5$ ppm corresponding to the presence of $\mathrm{CH}_{3}$ group, one singlet at $\delta 3.33 \mathrm{ppm}$ corresponding to the $\mathrm{NH}_{2}$ group, two multiplets at $\delta 7.00-7.71 \mathrm{ppm}$ corresponding to $\mathrm{C}_{6} \mathrm{H}_{5}$ group and a singlet at $\delta$ $9.54 \mathrm{ppm}$ for the $\mathrm{NH}$ group. Moreover, the ${ }^{13} \mathrm{C}$ NMR data revealed the presence of signals at $16.9\left(\mathrm{CH}_{3}\right), 116.3(\mathrm{CN}), 119.0,120.2,123.8,125.5,134.2,139.6,143.2,144.1\left(\mathrm{C}_{6} \mathrm{H}_{5}\right.$, thiophene C), $163.9(\mathrm{CO})$.

The 4-methyl group present in compound $\mathbf{3}$ showed interesting reactivity due to the effect of the electron withdrawal effect of the o-cyano group. Thus, compound $\mathbf{3}$ coupled with the aryldiazonium salts 4a-d to give the aryl hydrazone derivatives 5a-d, respectively. The analytical and spectral data of 5a-d were the basis of their structure elucidation. On the other hand compound 3 reacted with benzaldehyde $\mathbf{6}$ to give the benzal derivative 7. Compound 3 reacted with acetic acid/acetic anhydride (3:1) to give the $2-\mathrm{N}$-acetyl derivative 8 . The analytical and spectral data of the latter product were in agreement with its structure. Thus, ${ }^{1} \mathrm{H}$ NMR spectrum of 8 showed two singlet at $\delta 2.50$ and $2.51 \mathrm{ppm}$ corresponding to the presence of two $\mathrm{CH}_{3}$ groups, one multiplets at $\delta 7.05-7.66 \mathrm{ppm}$ corresponding to the $\mathrm{C}_{6} \mathrm{H}_{5}$ group and two singlets at $\delta 10.00,11.96 \mathrm{ppm}$ corresponding to the presence of two $\mathrm{NH}$ group. Moreover, ${ }^{13} \mathrm{C}$ NMR showed the presence of signals at 19.6, $20.3\left(2 \mathrm{CH}_{3}\right),(\mathrm{C}=\mathrm{C}), 116.3(\mathrm{CN}), 123.2,124.8$, $125.3,126.9,128.9,130.2,132.7,134.6,142.2,143.4\left(\mathrm{C}_{6} \mathrm{H}_{5}\right.$, thiophene $\left.\mathrm{C}\right), 164.3,165.8$ (2CO).

Compound $\mathbf{8}$ coupled with the aromatic diazonium salts $\mathbf{4 a - d}$ to give the arylhydrazone derivatives 9a-d (Scheme 1). Encouraged by the excellent results, we next investigated the reactivity of compound 3 towards phenylisothiocyanate 10, where the reaction afforded the theino $[d]$ pyrimidine derivative 12 . The reaction took place through the intermediate formation of the N-phenylthiourea derivative 11. The structure of compound $\mathbf{1 2}$ was confirmed on the basis of analytical and spectral data. Thus, the ${ }^{1} \mathrm{H}$ NMR spectrum showed a singlet at $\delta 2.51$ ppm for the $\mathrm{CH}_{3}$ group, a multiplet at $\delta 7.30-7.66$ ppm corresponding to the two $\mathrm{C}_{6} \mathrm{H}_{5}$ groups, a singlet at $\delta 7.71 \mathrm{ppm}$ for SH group and two singlets at $\delta 9.54,11.08 \mathrm{ppm}$ corresponding to the presence of two $\mathrm{NH}$ group. Compound 3 reacted with elemental sulfur in the presence of triethylamine to give the thieno[c]thiophene derivative 14. The reaction took place through the first reaction of the $\mathrm{CH}_{3}$ group with elemental sulfur to give the thiol derivative $\mathbf{1 3}$ followed by the addition of the $\mathrm{SH}$ group to the $\mathrm{CN}$ group to give $\mathbf{1 4}$ (Scheme 2). On the other hand, the reaction of compound 3 with malononitrile (15) in 1,4-dioxane containing a catalytic amount of triethylamine gave the thieno[b]pyridine derivative 17. Formation of the latter product took place through the intermediate formation of the acyclic intermediate 16. The 2-amino group present in $\mathbf{3}$ showed interesting reactivity towards diazodization and coupling. Thus, compound 3 reacted with a cold solution of sodium nitrite in the presence of acetic acid/hydrochloric acid $(3: 1)$ to give the intermediate diazonium salt $\mathbf{1 8}$. The latter reacted with acetyl acetone $\mathbf{1 9}$ to give the hydrazone derivative 20 (Scheme 3). The analytical and spectral data of the latter product were the basis of its structure elucidation. Thus the ${ }^{1} \mathrm{H}$ NMR spectrum showed three singlets at $\delta 2.32,2.45,2.62$ ppm corresponding to the presence of three $\mathrm{CH}_{3}$ groups, a multiplet at $\delta 7.27-7.62 \mathrm{ppm}$ corresponding to the $\mathrm{C}_{6} \mathrm{H}_{5}$ group and two singlets at $\delta 7.72,9.54 \mathrm{ppm}$ for the two NH groups. In addition, the ${ }^{13} \mathrm{C}$ NMR spectrum showed the presence of signals at 19.8 , 20.6, $21.3\left(3 \mathrm{CH}_{3}\right), 117.0(\mathrm{CN}), 120.4,121.7,122.9,124.7,125.9,128.2,130.1,138.0\left(\mathrm{C}_{6} \mathrm{H}_{5}\right.$, thiophene C), 163.2, 164.0, $165.2(3 \mathrm{CO}), 173.2(\mathrm{C}=\mathrm{N})$. 
Uses of acetoacetanilide for the synthesis of thiophene derivatives with cytotoxic activities 521

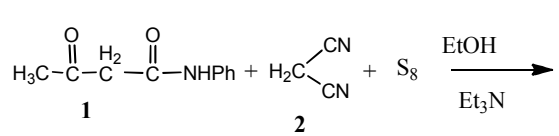<smiles>Cc1c(C(=O)Nc2ccccc2)sc(N)c1C#N</smiles>
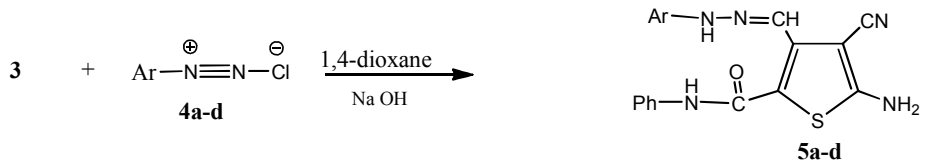

\begin{tabular}{|c|c|}
\hline $\mathbf{4}$ & $\mathrm{Ar}$ \\
\hline $\mathbf{a}$ & $\mathrm{Ph}$ \\
\hline $\mathbf{b}$ & $4-\mathrm{CH}_{3} \mathrm{C}_{6} \mathrm{H}_{4}$ \\
\hline $\mathbf{c}$ & $4-\mathrm{OCH}_{3} \mathrm{C}_{6} \mathrm{H}_{4}$ \\
\hline $\mathbf{d}$ & $\mathrm{ClC}_{6} \mathrm{H}_{4}$ \\
\hline
\end{tabular}

\begin{tabular}{|c|c|}
\hline $\mathbf{5}$ & $\mathrm{Ar}$ \\
\hline $\mathbf{a}$ & $\mathrm{Ph}$ \\
\hline $\mathbf{b}$ & $4-\mathrm{CH}_{3} \mathrm{C}_{6} \mathrm{H}_{4}$ \\
\hline $\mathbf{c}$ & $4-\mathrm{OCH}_{3} \mathrm{C}_{6} \mathrm{H}_{4}$ \\
\hline $\mathbf{d}$ & $\mathrm{ClC}_{6} \mathrm{H}_{4}$ \\
\hline
\end{tabular}
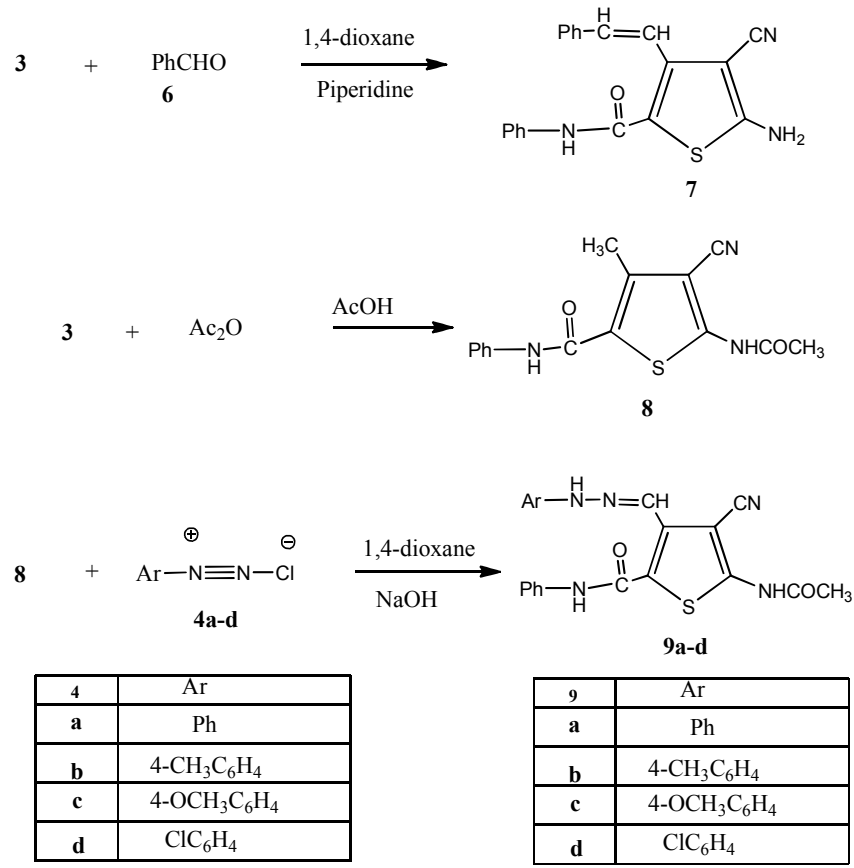

Scheme 1

Bull. Chem. Soc. Ethiop. 2017, 31(3) 
<smiles>Cc1c(C(=O)Nc2ccccc2)sc(N)c1C#N</smiles>

$\mathrm{PhNCS}$

10<smiles>CCCNC(=O)NCCCc1sc(NC(=S)Nc2ccccc2)c(C)c1C#N</smiles><smiles>Cc1c(C(=O)Nc2ccccc2)sc2nc(S)n(-c3ccccc3)c(=N)c12</smiles>

12<smiles>N#Cc1c(N)sc(C(=O)Nc2cccs2)c1CS</smiles><smiles>N=C1SCc2c(C(=O)Nc3ccccc3)sc(N)c21</smiles>

14

Scheme 2

Thus, compound $\mathbf{3}$ reacted with ethyl cyanoacetate $\mathbf{2 1}$ in refluxing dimethylformamide to give the amide derivative $\mathbf{2 2}$. The analytical and spectral data of compound $\mathbf{2 2}$ are in agreement with its structure. The reaction of $\mathbf{2 2}$ with benzaldehyde $\mathbf{6}$ gave the benzylidene derivative $\mathbf{2 3}$. Moreover, the reaction of $\mathbf{2 2}$ with salicyladehyde $\mathbf{2 4}$ gave the coumarin derivative $\mathbf{2 5}$. The excellent yield of compound $\mathbf{2 2}$ encouraged us to study its reactivity towards aromatic diazonium salts in the aim of synthesizing new aryl hydrazine derivatives with different cytotoxic activities. Thus, the reaction of $\mathbf{2 2}$ with aromatic diazonium salts $\mathbf{4 a - d}$ gave the arylhydrazone derivatives $\mathbf{2 6 a - d}$. The analytical and spectral data of the latter products were the basis of their structure elucidation. Finally the reaction of compound $\mathbf{2 2}$ with elemental sulfur and malononitrile 15 gave the thiophene derivative 27 (Scheme 4). 
Uses of acetoacetanilide for the synthesis of thiophene derivatives with cytotoxic activities 523

3
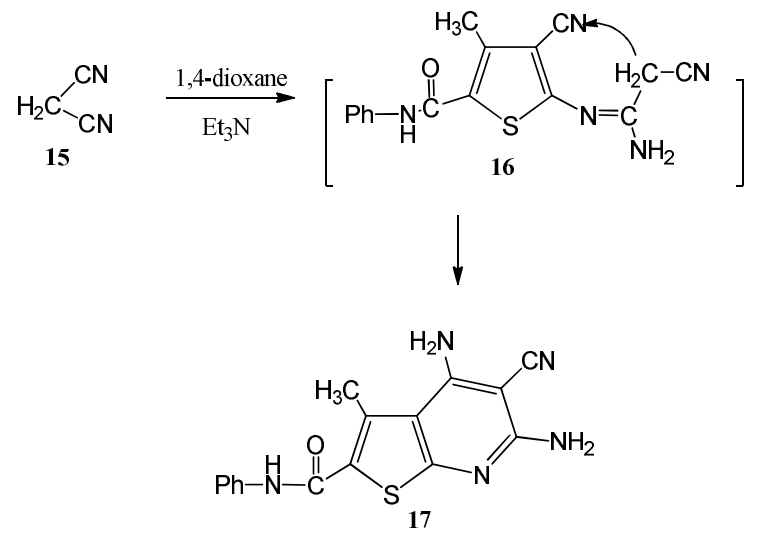

$3+\mathrm{NaNO}_{2} \stackrel{\mathrm{HCl}}{\longrightarrow}$

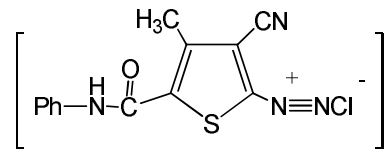

18
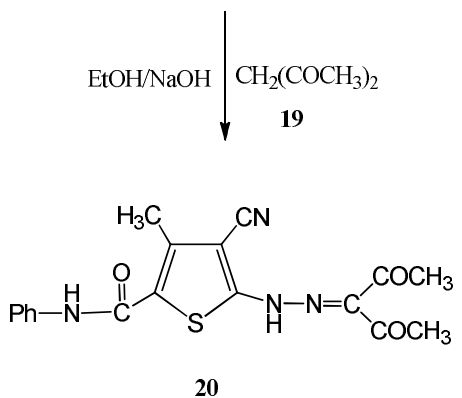

Scheme 3 


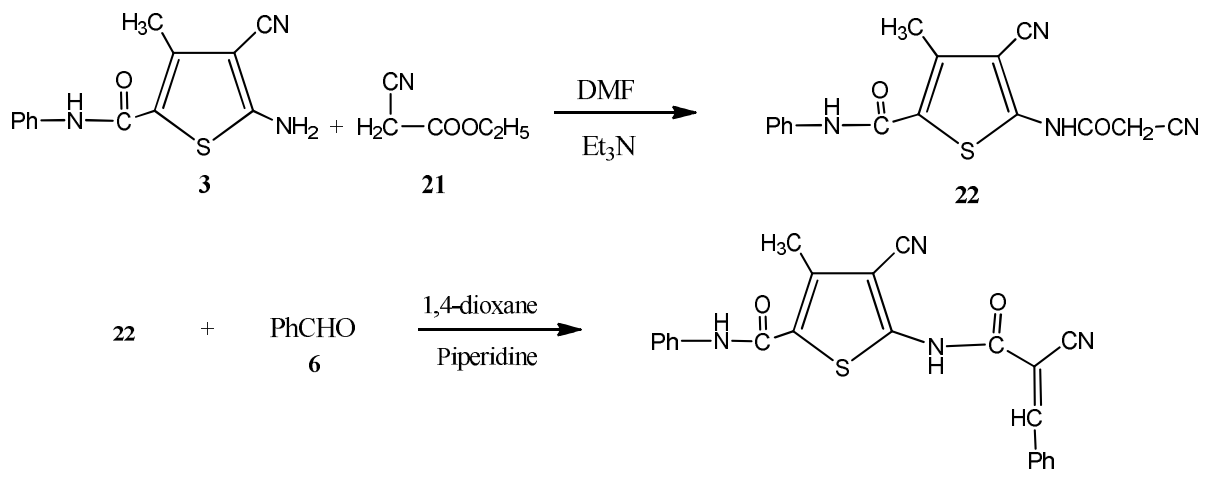

23

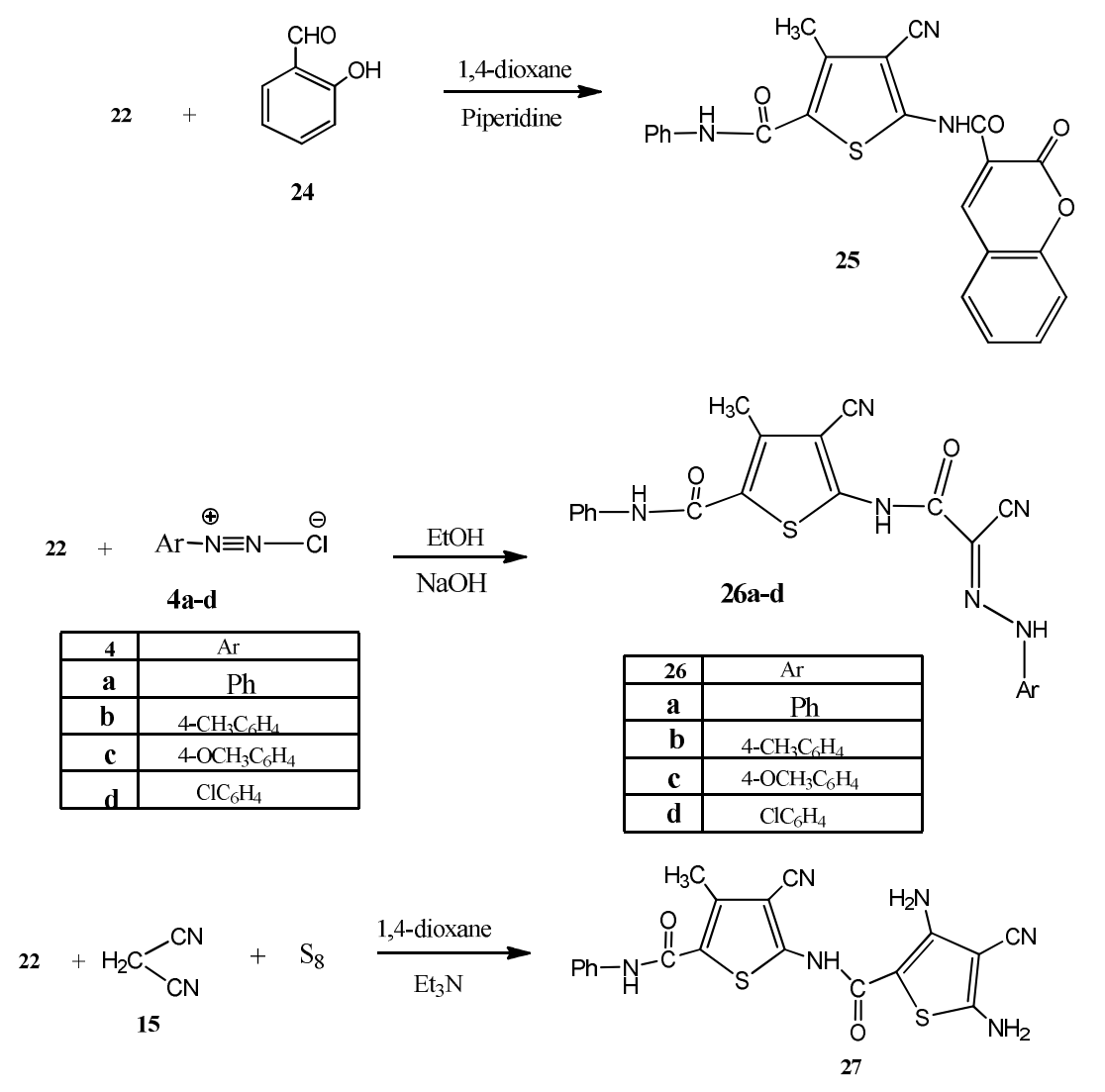

Scheme 4 


\section{Structure activity relationship}

From Table 1 it is clear that the benzimidazole moiety was found to be crucial for the cytotoxic effect of the cyclic compounds 3 to 27. Compounds $5 \mathbf{a}, \mathbf{5 b}, \mathbf{8}, \mathbf{2 2}$, and 23 exhibited optimal cytotoxic effect against cancer cell lines. On the other hand compound $\mathbf{9 c}, \mathbf{2 6 d}$ and 27 showed very low activities toward the three cancer cell lines. Compounds 3, 5c, 5d, 7, 9d, 14, 20, 25 and 26c showed moderate activities. The thiophene derivative 3 showed low potency, however its reaction with difference aryl diazonium salts gave the arylhydrazone derivatives 5a-c. It is obvious that compounds $\mathbf{5 a}(\mathrm{R}=\mathrm{H})$ and $\mathbf{5 b}\left(\mathrm{R}=\mathrm{CH}_{3}\right)$ showed the highest cytotoxicity among the three compounds. The reaction of compound $\mathbf{3}$ with benzaldehyde produced compound 7 with low potency. The thiophene derivative 7 showed low potency although its acetylation gave compound $\mathbf{8}$ with high potency against the three cancer cell lines. For the thiophenes derivatives 9a-d, it is clear that compound $\mathbf{9 b}$ with 6- the 4-methylphenyl group showed the highest activity among the four compounds which is considered as a moderate activity. Compound $\mathbf{1 2}$ which was a thieno $[d]$ pyrimidine derivative showed highest activity among the tested compounds. Similarly, compound $\mathbf{1 7}$ which is a thieno $[b]$ pyridine derivative showed high potency towards the three cancer cell lines. Compounds 22, 23, 26a and 26b showed relatively high potency and such activity is attributed to the presence of the N-acyl moieties. From the activity of the compounds towards the three cancer cell lines it is obvious that the presence of N-rich and Scontaining heterocyclic ring was the main effect through the high potency of the compound.

Table 1. Effect of the newly synthesized compounds on the growth of three human tumor cell lines $\mathrm{GI}_{50}\left(\mathrm{mmol} \mathrm{L}^{-1}\right)$.

\begin{tabular}{|c|c|c|c|}
\hline Compound No. & MCF-7 & NCI-H460 & SF-268 \\
\hline 3 & $22.6 \pm 1.4$ & $24.9 \pm 2.8$ & $13.8 \pm 3.8$ \\
\hline $5 \mathbf{a}$ & $0.4 \pm 0.2$ & $0.1 \pm 0.02$ & $0.9 \pm 0.08$ \\
\hline $5 \mathbf{b}$ & $0.01 \pm 0.002$ & $0.01 \pm 0.004$ & $0.04 \pm 0.01$ \\
\hline $5 c$ & $30.6 \pm 10.2$ & $32.6 \pm 8.6$ & $24.4 \pm 12.8$ \\
\hline $5 d$ & $18.1 \pm 1.6$ & $6.2 \pm 2.8$ & $8.6 \pm 2.6$ \\
\hline 7 & $28.6 \pm 12.2$ & $12.6 \pm 8.6$ & $52.4 \pm 14.6$ \\
\hline 8 & $0.7 \pm 0.50$ & $0.2 \pm 0.08$ & $1.0 \pm 0.02$ \\
\hline $9 \mathbf{a}$ & $70.7 \pm 18.5$ & $40.2 \pm 12.8$ & $52.4 \pm 8.6$ \\
\hline $9 \mathrm{~b}$ & $10.8 \pm 2.6$ & $4.5 \pm 0.8$ & $4.8 \pm 1.8$ \\
\hline $9 \mathrm{c}$ & $60.6 \pm 16.9$ & $38.9 \pm 10.8$ & $50.8 \pm 8.6$ \\
\hline 9d & $26.4 \pm 2.2$ & $34.1 \pm 0.8$ & $18.8 \pm 4.8$ \\
\hline 12 & $0.01 \pm 0.006$ & $0.03 \pm 0.001$ & $0.02 \pm 0.004$ \\
\hline 14 & $18.1 \pm 0.6$ & $16.3 \pm 1.4$ & $12.3 \pm 1.5$ \\
\hline 17 & $0.06 \pm 0.004$ & $0.03 \pm 0.003$ & $0.1 \pm 0.02$ \\
\hline 20 & $32.8 \pm 0.6$ & $36.5 \pm 0.8$ & $30.7 \pm 1.6$ \\
\hline 22 & $0.3 \pm 0.1$ & $0.2 \pm 0.08$ & $0.5 \pm 0.01$ \\
\hline 23 & $0.9 \pm 0.2$ & $0.1 \pm 0.02$ & $0.3 \pm 0.05$ \\
\hline 25 & $22.6 \pm 2.6$ & $24.3 \pm 0.8$ & $30.9 \pm 3.8$ \\
\hline $26 a$ & $0.01 \pm 0.003$ & $0.02 \pm 0.001$ & $0.01 \pm 0.001$ \\
\hline $26 b$ & $0.02 \pm 0.008$ & $0.03 \pm 0.006$ & $0.05 \pm 0.00$ \\
\hline $26 \mathrm{c}$ & $22.4 \pm 2.2$ & $32.6 \pm 1.4$ & $26.8 \pm 6.4$ \\
\hline 26d & $55.1 \pm 2.7$ & $23.2 \pm 4.8$ & $14.4 \pm 2.6$ \\
\hline 27 & $36.0 \pm 1.8$ & $43.0 \pm 0.8$ & $30.5 \pm 1.1$ \\
\hline Doxorubicin & $0.04 \pm 0.008$ & $0.09 \pm 0.008$ & $0.09 \pm 0.007$ \\
\hline
\end{tabular}

Results are given in concentrations that were able to cause $50 \%$ of cell growth inhibition $\left(\mathrm{GI}_{50}\right)$ after a continuous exposure of $48 \mathrm{~h}$ and show means \pm SEM of three-independent experiments performed in duplicate. 


\section{EXPERIMENTAL}

\section{Antitumor and normal cell line}

Reagents. Fetal bovine serum (FBS) and L-glutamine were from Gibco Invitrogen Co. (Scotland, UK). RPMI-1640 medium was from Cambrex (New Jersey, USA). Dimethyl sulfoxide (DMSO), doxorubicin, penicillin, streptomycin and sulforhodamine B (SRB) were from Sigma Chemical Co. (Saint Louis, USA).

Cell cultures. Three human tumor cell lines, MCF-7 (breast adenocarcinoma), NCI-H460 (nonsmall cell lung cancer), and SF-268 (CNS cancer) were used. MCF-7 was obtained from the European Collection of Cell Cultures (ECACC, Salisbury, UK) and NCI-H460 and SF-268 were kindly provided by the National Cancer Institute (NCI, Cairo, Egypt). They grow as monolayer and routinely maintained in RPMI-1640 medium supplemented with 5\% heat inactivated FBS, 2 $\mathrm{mM}$ glutamine and antibiotics (penicillin $100 \mathrm{U} / \mathrm{mL}$, streptomycin $100 \mathrm{mg} / \mathrm{mL}$ ), at $37{ }^{\circ} \mathrm{C}$ in a humidified atmosphere containing $5 \% \mathrm{CO}_{2}$. Exponentially growing cells were obtained by plating $1.5 \times 10^{5}$ cells $/ \mathrm{mL}$ for MCF-7 and SF-268 and $0.75 \times 10^{4}$ cells $/ \mathrm{mL}$ for NCI-H460, followed by $24 \mathrm{~h}$ of incubation. The effect of the vehicle solvent (DMSO) on the growth of these cell lines was evaluated in all the experiments by exposing untreated control cells to the maximum concentration $(0.5 \%)$ of DMSO used in each assay.

Tumor cell growth assay. The effects of 3-27 on the in vitro growth of human tumor cell lines were evaluated according to the procedure adopted by the National Cancer Institute (NCI, USA) in the 'In vitro Anticancer Drug Discovery Screen' that uses the protein-binding dye sulforhodamine B to assess cell growth. Briefly, exponentially, cells growing in 96-well plates were then exposed for $48 \mathrm{~h}$ to five serial concentrations of each compound, starting from a maximum concentration of $150 \mu \mathrm{M}$. Following this exposure period adherent cells were fixed, washed, and stained. The bound stain was solubilized and the absorbance was measured at 492 $\mathrm{nm}$ in a plate reader (Bio-Tek Instruments Inc., Powerwave XS, Wincoski, USA). For each test compound and cell line, a dose-response curve was obtained and the growth inhibition of $50 \%$ $\left(\mathrm{GI}_{50}\right)$, corresponding to the concentration of the compounds that inhibited $50 \%$ of the net cell growth was calculated as described elsewhere. Doxorubicin was used as a positive control and tested in the same manner.

\section{Instruments}

All melting points are uncorrected. IR spectra were recorded for $\mathrm{KBr}$ discs on a PyeUnicam SP1000 spectrophotometer. ${ }^{1} \mathrm{H}$ NMR spectra were measured on a Varian EM-390-200 MHz in DMSO as solvent using TMS as internal standard and chemical shifts are expressed as $\delta$. Analytical data were obtained from the Microanalytical Data Unit at Cairo University, Giza, Egypt.

General procedure for the synthesis of 5-amino-4-cyano-3-methyl-N-phenylthiophene-2carboxamide 3

To a solution of 3-oxo-N-phenylbutanamide $(17.7 \mathrm{~g}, 0.1 \mathrm{~mol})$ in absolute ethanol $(50 \mathrm{~mL})$ containing triethylamine $(2.0 \mathrm{~mL})$, malononitrile $(6.6 \mathrm{~g}, 0.1 \mathrm{~mol})$ and sulfur $(3.2 \mathrm{~g}, 0.1 \mathrm{~mol})$ was added. The reaction mixture was heated under reflux for $3 \mathrm{~h}$ then left to cool. The formed solid produce was collected by filtration and dried. The obtained product was crystallized from ethanol to give pale yellow crystals. Yield $20.5 \mathrm{~g}(80 \%)$; m.p. $225{ }^{\circ} \mathrm{C}$; anal. calcd for $\mathrm{C}_{13} \mathrm{H}_{11} \mathrm{~N}_{3} \mathrm{OS}$ (257.06): C, 60.68; H, 4.31; N, 16.33; S, 12.46\%; found: C, 60.76; H, 4.47; N, 
Uses of acetoacetanilide for the synthesis of thiophene derivatives with cytotoxic activities 527

16.60; S, 12.51\%. IR (KBr) v/cm $\mathrm{cm}^{-1} 3466-3320\left(\mathrm{NH}_{2}, \mathrm{NH}\right), 3055(\mathrm{CH}$ aromatic), $2220(\mathrm{CN})$, $1688(\mathrm{CO}), 1630(\mathrm{C}=\mathrm{C}) .{ }^{1} \mathrm{H}$ NMR $\left(\mathrm{DMSO}_{6}\right) \delta 2.50\left(\mathrm{~s}, 3 \mathrm{H}, \mathrm{CH}_{3}\right), 3.33\left(\mathrm{~s}, 2 \mathrm{H}, \mathrm{NH}_{2}\right), 7.00-7.71$ $\left(\mathrm{m}, 5 \mathrm{H}, \mathrm{C}_{6} \mathrm{H}_{5}\right), 9.54(\mathrm{~s}, 1 \mathrm{H}, \mathrm{NH}) .{ }^{13} \mathrm{C} \mathrm{NMR}\left(\mathrm{DMSO}_{-} \mathrm{d}_{6}\right) \delta 16.9\left(\mathrm{CH}_{3}\right), 116.3(\mathrm{CN}), 119.0,120.2$, $123.8,125.5,134.2,139.6,143.2,144.1\left(\mathrm{C}_{6} \mathrm{H}_{5}\right.$, thiophene $\left.\mathrm{C}\right), 163.9(\mathrm{CO})$.

General procedure for the synthesis of the thiophenederivatives $\mathbf{5 a - d}$

To a cold solution of $\mathbf{3}(2.57 \mathrm{~g}, 0.01 \mathrm{~mol})$ in 1,4-dioxane $(40 \mathrm{~mL})$ containing sodium hydroxide $(2.5 \mathrm{~g})$ a cold solution of the respective diazonium salt [prepared by the addition of sodium nitrite solution $(0.70 \mathrm{~g}, 0.01 \mathrm{~mol})$ to a cold solution of either aniline $(0.94 \mathrm{~g}, 0.01 \mathrm{~mol}), p$ toluidine (1.15 g, $0.01 \mathrm{~mol}), p$-methoxy aniline $(1.3 \mathrm{~g}, 0.01 \mathrm{~mol})$ or $p$-chloroaniline $(1.29 \mathrm{~g}, 0.01$ $\mathrm{mol})$ in concentrated hydrochloric acid $(12 \mathrm{~mL})$ with continuous stirring] was added while stirring. The formed solid product, in each case, upon stirring at room temperature for $1 \mathrm{~h}$ was collected by filtration and dried. The obtained product was crystallized from ethanol to give brown crystals.

5-Amino-4-cyano-N-phenyl-3-((2-phenylhydrazono) methyl) thiophene-2-carboxamide (5a). Yield 2.7 g (75\%); m.p. 135-140 ${ }^{\circ} \mathrm{C}$; anal. calcd. for $\mathrm{C}_{19} \mathrm{H}_{15} \mathrm{~N}_{5} \mathrm{OS}$ (361.42): C, 63.14; H, 4.18; N, 19.38; S, 8.87\%; found: C, 63.06; H, 4.27; N, 19.44; S, 8.91\%. IR (KBr) v/cm $\mathrm{cm}^{-1} 3472-3328$ $\left(2 \mathrm{NH}, \mathrm{NH}_{2}\right), 3055\left(\mathrm{CH}\right.$ aromatic), $2220(\mathrm{CN}), 1687(\mathrm{CO}), 1656(\mathrm{C}=\mathrm{N}), 1628(\mathrm{C}=\mathrm{C}) .{ }^{1} \mathrm{H}$ NMR $\left(\mathrm{DMSO}_{-} \mathrm{d}_{6}\right) \delta 3.33\left(\mathrm{~s}, 2 \mathrm{H}, \mathrm{NH}_{2}\right), 6.39(\mathrm{~s}, 1 \mathrm{H}, \mathrm{CH}), 6.40(\mathrm{~s}, 1 \mathrm{H}, \mathrm{NH}), 7.05-7.71\left(\mathrm{~m}, 10 \mathrm{H}, 2 \mathrm{C}_{6} \mathrm{H}_{5}\right)$, $9.54(\mathrm{~s}, 1 \mathrm{H}, \mathrm{NH}) .{ }^{13} \mathrm{C}$ NMR $\left(\mathrm{DMSO}_{-} \mathrm{d}_{6}\right) \delta 116.6(\mathrm{CN}), 119.8,121.6,124.3,125.8,128.0,129.6$, 130.2, 134.5, 139.8, 143.8, 144.7 (two $\mathrm{C}_{6} \mathrm{H}_{5}$, thiophene $\left.\mathrm{C}\right), 164.2(\mathrm{CO}), 172.1(\mathrm{C}=\mathrm{N})$.

5-Amino-4-cyano- $N$-phenyl-3-((2-(p-tolyl) hydrazono) methyl) thiophene-2-carboxamide (5b). Yield 2.62 g (70\%); m.p. 140-150 ${ }^{\circ} \mathrm{C}$; anal. calcd. for $\mathrm{C}_{20} \mathrm{H}_{17} \mathrm{~N}_{5} \mathrm{OS}$ (375.54): C, 63.98; H, 4.56; $\mathrm{N}, 18.65 ; \mathrm{S}, 8.54 \%$; found: C, 63.79; H, 4.31; N, 18.72; S, 8.76\%. IR (KBr) v/cm $\mathrm{cm}^{-1} 3477-3318$ $\left(\mathrm{NH}_{2}, 2 \mathrm{NH}\right), 3062\left(\mathrm{CH}\right.$ aromatic), $2223(\mathrm{CN}), 1688(\mathrm{CO}), 1660(\mathrm{C}=\mathrm{N}), 1628(\mathrm{C}=\mathrm{C}) .{ }^{1} \mathrm{H}$ NMR $\left(\mathrm{DMSO}_{-} \mathrm{d}_{6}\right) \delta 2.29\left(\mathrm{~s}, 3 \mathrm{H}, \mathrm{CH}_{3}\right), 3.30\left(\mathrm{~s}, 2 \mathrm{H}, \mathrm{NH}_{2}\right), 7.05(\mathrm{~s}, 1 \mathrm{H}, \mathrm{CH}), 7.12(\mathrm{~s}, 1 \mathrm{H}, \mathrm{NH}), 7.32-7.71$ $\left(\mathrm{m}, 9 \mathrm{H}, \mathrm{C}_{6} \mathrm{H}_{4}, \mathrm{C}_{6} \mathrm{H}_{5}\right), 9.54(\mathrm{~s}, 1 \mathrm{H}, \mathrm{NH}){ }^{13} \mathrm{C}$ NMR (DMSO-d $\left.)_{6}\right) \delta 19.8\left(\mathrm{CH}_{3}\right), 116.6(\mathrm{CN}), 119.3$, $122.5,124.6,125.2$, 128.3, 129.8, 131.4, 132.9, 138.4, 142.7, $143.9\left(\mathrm{C}_{6} \mathrm{H}_{5}, \mathrm{C}_{6} \mathrm{H}_{4}\right.$, thiophene $\left.\mathrm{C}\right)$.

5-Amino-3-((2-(4-methoxyphenyl)hydrazono)methyl)-4-cyano- $N$-phenylthiophene-2-carboxamide (5c). Yield $3.24 \mathrm{~g} \mathrm{(83 \% );} \mathrm{m.p.} 120-125^{\circ} \mathrm{C}$; Anal. Calcd. for $\mathrm{C}_{20} \mathrm{H}_{17} \mathrm{~N}_{5} \mathrm{O}_{2} \mathrm{~S}$ (391.45): C, 61.37; H, 4.38; N, 17.89;S, 8.19\%; found: C, 61.43; H, 4.23; N, 18.02; S, 8.22\%. IR (KBr) v/cm 1 3484-3322 $\left(\mathrm{NH}_{2}, 2 \mathrm{NH}\right), 3062(\mathrm{CH}$ aromatic), $2221(\mathrm{CN}), 1689(\mathrm{CO}), 1662(\mathrm{C}=\mathrm{N}), 1625$ $(\mathrm{C}=\mathrm{C}) .{ }^{1} \mathrm{H}$ NMR $\left(\mathrm{DMSO}_{-} \mathrm{d}_{6}\right) \delta 3.74\left(\mathrm{~s}, 2 \mathrm{H}, \mathrm{NH}_{2}\right), 3.75\left(\mathrm{~s}, 3 \mathrm{H}, \mathrm{CH}_{3}\right), 6.93(\mathrm{~s}, 1 \mathrm{H}, \mathrm{CH}), 7.01(\mathrm{~s}, 1 \mathrm{H}$, $\mathrm{NH}), 7.30-7.67\left(\mathrm{~m}, 9 \mathrm{H}, \mathrm{C}_{6} \mathrm{H}_{5}, \mathrm{C}_{6} \mathrm{H}_{4}\right), 9.50(\mathrm{~s}, 1 \mathrm{H}, \mathrm{NH}) .{ }^{13} \mathrm{C}$ NMR $\left(\mathrm{DMSO}-\mathrm{d}_{6}\right) \delta 19.3\left(\mathrm{CH}_{3}\right)$, $116.3(\mathrm{CN}), 119.9,123.2,124.9,125.1,128.6,129.1,132.0,132.9,138.4,142.7,143.4\left(\mathrm{C}_{6} \mathrm{H}_{5}\right.$, $\mathrm{C}_{6} \mathrm{H}_{4}$, thiophene $\left.\mathrm{C}\right), 164.8(\mathrm{CO}), 171.2(\mathrm{C}=\mathrm{N})$.

5-Amino-3-((2-(4-chlorophenyl)hydrazono)methyl)-4-cyano- $N$-phenylthiophene-2-carboxamide (5d). Yield $3.36 \mathrm{~g}(85 \%)$; m.p. $120{ }^{\circ} \mathrm{C}$; anal. calcd. for $\mathrm{C}_{19} \mathrm{H}_{14} \mathrm{ClN}_{5} \mathrm{OS}(395.87): \mathrm{C}, 57.65 ; \mathrm{H}$, 3.56 ; N, 17.69; S, 8.10\%; found: C, 57.90; H, 3.47; N, 17.88; S, 8.28\%. IR (KBr) v/cm ${ }^{-1} 3489$ $3328\left(\mathrm{NH}_{2}, 2 \mathrm{NH}\right), 3051\left(\mathrm{CH}\right.$ aromatic), $2220(\mathrm{CN}), 1687(\mathrm{CO}), 1665(\mathrm{C}=\mathrm{N}), 1621(\mathrm{C}=\mathrm{C}) .{ }^{1} \mathrm{H}$ NMR $\left(\mathrm{DMSO}_{-} \mathrm{d}_{6}\right) \delta 3.81\left(\mathrm{~s}, 2 \mathrm{H}, \mathrm{NH}_{2}\right), 6.90(\mathrm{~s}, 1 \mathrm{H}, \mathrm{CH}), 7.02(\mathrm{~s}, 1 \mathrm{H}, \mathrm{NH}), 7.30-7.67(\mathrm{~m}, 9 \mathrm{H}$, $\left.\mathrm{C}_{6} \mathrm{H}_{4}, \mathrm{C}_{6} \mathrm{H}_{5}\right), 9.52(\mathrm{~s}, 1 \mathrm{H}, \mathrm{NH}) .{ }^{13} \mathrm{C}$ NMR $\left(\mathrm{DMSO}_{-} \mathrm{d}_{6}\right) \delta 116.4(\mathrm{CN}), 119.9,123.8,124.9,125.4$, 128.1, 128.4, 132.3, 132.6, 134.6, 142.9, $143.5\left(\mathrm{C}_{6} \mathrm{H}_{5}, \mathrm{C}_{6} \mathrm{H}_{4}\right.$, thiophene C), 164.1 (CO), 171.1 $(\mathrm{C}=\mathrm{N})$. 
To a solution of $3(2.57 \mathrm{~g}, 0.01 \mathrm{~mol})$ in 1,4-dioxane $(40 \mathrm{~mL})$ containing piperidine $(1.0 \mathrm{~mL})$, benzaldehyde $(1.0 \mathrm{~g}, 0.01 \mathrm{~mol})$ was added. The reaction mixture, in each case, was heated under reflux for $8 \mathrm{~h}$ then left to cool. The solid product formed upon evaporating the solution under vacuum followed by triturating the remaining product with ethanol, was collected by filtration and dried. The obtained product was crystallized from ethanol to give pale brown crystals. Yield 2.76 g (80\%); m.p. $180{ }^{\circ} \mathrm{C}$; anal. calcd. for $\mathrm{C}_{20} \mathrm{H}_{15} \mathrm{~N}_{3} \mathrm{OS}$ (345.42): C, 69.54; H, 4.38; N, 12.17; S, 9.28\%; found: C, 69.69; H, 4.29; N, 12.28; S, 9.41\%; IR (KBr) v/cm $\mathrm{cm}^{-1} 3426-3365\left(\mathrm{NH}_{2}, \mathrm{NH}\right)$, $3054\left(\mathrm{CH}\right.$, aromatic), $2219(\mathrm{CN}), 1801(\mathrm{C}=\mathrm{O}), 1660(\mathrm{C}=\mathrm{N}) ;{ }^{1} \mathrm{H}$ NMR $\left(\mathrm{DMSO}_{6}\right) \delta 3.31(\mathrm{~s}, 2 \mathrm{H}$, $\left.\mathrm{NH}_{2}\right), 7.05-7.27(2 \mathrm{~d}, 2 \mathrm{H}, \mathrm{CH}=\mathrm{CH}), 7.30-7.59\left(\mathrm{~m}, 10 \mathrm{H}, 2 \mathrm{C}_{6} \mathrm{H}_{5}\right), 9.54(\mathrm{~s}, 1 \mathrm{H}, \mathrm{NH}),{ }^{13} \mathrm{C} \mathrm{NMR}$ $\left(\right.$ DMSO $\left._{6}\right) \delta 90.6,98.3(\mathrm{C}=\mathrm{C}), 116.5(\mathrm{CN}), 120.4,122.6,124.6,126.9,128.3,128.9,132.1$, $132.8,134.6,142.9,143.9$ (two $\mathrm{C}_{6} \mathrm{H}_{5}$, thiophene $\mathrm{C}$ ), 164.13 (CO).

\section{5-Acetamido-4-cyano-3-methyl-N-phenylthiophene-2-carboxamide (8)}

To a solution of $3(2.57 \mathrm{~g}, 0.01 \mathrm{~mol})$ in acetic acid $(30 \mathrm{~mL})$, acetic anhydride $(1.02 \mathrm{~g}, 0.01 \mathrm{~mol})$ was added. The reaction mixture was heated under reflux for $3 \mathrm{~h}$ then left to cool. The solid product formed upon pouring onto ice/water containing a few drops of hydrochloric acid was collected by filtration and dried. The obtained product was crystallized from ethanol to give pale yellow crystals. Yield 2.33 g (78\%), m.p. $260{ }^{\circ} \mathrm{C}$. anal. calcd. for $\mathrm{C}_{15} \mathrm{H}_{13} \mathrm{~N}_{3} \mathrm{O}_{2} \mathrm{~S}$ (299.35): C, 60.18; H, 4.38; N, 14.04; S, 10.71\%; found: C, 62.42; H, 4.36; N, 14.11; S, 10.84\%. IR (KBr) v/cm ${ }^{-1} 3446-3268\left(\mathrm{NH}_{2}, 2 \mathrm{NH}\right), 3051(\mathrm{CH}$, aromatic $), 2221(\mathrm{CN}), 1703(\mathrm{C}=\mathrm{O}), 1643(\mathrm{C}=\mathrm{C}) .{ }^{1} \mathrm{H}$ NMR $\left(\right.$ DMSO-d $\left.{ }_{6}\right) \delta 2.50,2.51\left(2 \mathrm{~s}, 6 \mathrm{H}, 2 \mathrm{CH}_{3}\right), 7.05-7.66\left(\mathrm{~m}, 5 \mathrm{H}, \mathrm{C}_{6} \mathrm{H}_{5}\right), 10.00,11.96(2 \mathrm{~s}, 2 \mathrm{H}$, $2 \mathrm{NH}) .{ }^{13} \mathrm{C}$ NMR (DMSO-d $\left.\mathrm{d}_{6}\right) \delta 19.6,20.3\left(2 \mathrm{CH}_{3}\right),(\mathrm{C}=\mathrm{C}), 116.3(\mathrm{CN}), 123.2,124.8,125.3$, $126.9,128.9,130.2$, 132.7, 134.6, 142.2, $143.4\left(\mathrm{C}_{6} \mathrm{H}_{5}\right.$, thiophene $\left.\mathrm{C}\right), 164.3,165.8(2 \mathrm{CO})$.

General procedure for the synthesis of thiophene-2-carboxamidederivatives $\mathbf{9 a - d}$

To a cold solution of $8(2.99 \mathrm{~g}, 0.01 \mathrm{~mol})$ in ethanol $(40 \mathrm{~mL})$ containing sodium acetate $(2.5 \mathrm{~g})$ a cold solution of the respective diazonium salt [prepared by the addition of sodium nitrite solution $(0.70 \mathrm{~g}, 0.01 \mathrm{~mol})$ to a cold solution of either aniline $(0.94 \mathrm{~g}, 0.01 \mathrm{~mol}), p$-toluidine $(1.15 \mathrm{~g}, 0.01 \mathrm{~mol}), p$-methoxy aniline $(1.3 \mathrm{~g}, 0.01 \mathrm{~mol})$ or $p$-chloroaniline $(1.29 \mathrm{~g}, 0.01 \mathrm{~mol})$ in concentrated hydrochloric acid $(12 \mathrm{~mL})$ with continuous stirring] was added while stirring. The formed solid product, in each case, upon stirring at room temperature for $1 \mathrm{~h}$ was collected by filtration and dried. The obtained product was crystallized from ethanol to give yellowish brown crystals for $9 \mathrm{a}$ and $9 \mathrm{~b}$ and reddish yellow crystals for $9 \mathrm{c}$ and $9 \mathrm{~d}$.

5-Acetamido-4-cyano- $N$-phenyl-3-((2-phenylhydrazono)methyl) thiophene-2-carboxamide (9a). Yield $2.82 \mathrm{~g} \mathrm{(70 \% );} \mathrm{m.p.} \mathrm{over} 300{ }^{\circ} \mathrm{C}$; anal. calcd. for $\mathrm{C}_{21} \mathrm{H}_{17} \mathrm{~N}_{5} \mathrm{O}_{2} \mathrm{~S}$ (403.46): C, 62.52; $\mathrm{H}, 4.25$; N, 17.36; S, 7.95\%; found: C, 62.44; H, 4.11; N, 17.47; S, 8.05\%. IR (KBr) v/cm ${ }^{-1}: 3488-3328$ $(2 \mathrm{NH}), 3050\left(\mathrm{CH}\right.$ aromatic), $2220(\mathrm{CN}), 1685,1680(2 \mathrm{CO}), 1660(\mathrm{C}=\mathrm{N}), 1630(\mathrm{C}=\mathrm{C}) .{ }^{1} \mathrm{H}$ NMR $\left(\mathrm{DMSO}_{-} \mathrm{d}_{6}\right) \delta 2.26\left(\mathrm{~s}, 3 \mathrm{H}, \mathrm{CH}_{3}\right), 7.07(\mathrm{CH}=\mathrm{N}), 7.08(\mathrm{~s}, 1 \mathrm{H}, \mathrm{NH}), 7.12-7.66\left(\mathrm{~m}, 10 \mathrm{H}, 2 \mathrm{C}_{6} \mathrm{H}_{5}\right)$, 10.00, $11.96(2 \mathrm{~s}, 2 \mathrm{H}, 2 \mathrm{NH}) .{ }^{13} \mathrm{C}$ NMR $\left(\mathrm{DMSO}_{6}\right) \delta 19.8\left(\mathrm{CH}_{3}\right), 116.8(\mathrm{CN}), 119.4,121.7$, $122.0,123.8,124.9,130.3,133.8,134.2,138.5,140.4,144.2$ (two $\mathrm{C}_{6} \mathrm{H}_{5}$, thiophene $\mathrm{C}$ ), 163.8, $164.2(2 \mathrm{CO}), 172.3(\mathrm{C}=\mathrm{N})$.

5-Acetamido-4-cyano-N-phenyl-3-((2-(p-tolyl)hydrazono) methyl) thiophene-2-carboxamide (9b). Yield $3.33 \mathrm{~g}(80 \%)$; m.p. $290{ }^{\circ} \mathrm{C}$; anal. calcd. for $\mathrm{C}_{22} \mathrm{H}_{19} \mathrm{~N}_{5} \mathrm{O}_{2} \mathrm{~S}$ (417.48): C, 63.29; H, 4.59; N, 16.78; S, 7.68\%; found: C, 62.88; H, 4.33; N, 16.92; S, 7.82\%. IR (KBr) v/cm $\mathrm{cm}^{-1} 3447-3267$ (3NH), $3052\left(\mathrm{CH}\right.$, aromatic), $2970\left(\mathrm{CH}_{3}\right), 2223(\mathrm{CN}), 1704(\mathrm{C}=\mathrm{O}), 1643(\mathrm{C}=\mathrm{N}), 1529(\mathrm{C}=\mathrm{C}) ;{ }^{1} \mathrm{H}$ 
Uses of acetoacetanilide for the synthesis of thiophene derivatives with cytotoxic activities 529

NMR (DMSO-d $\left.\mathrm{d}_{6}\right) \delta 2.25,2.50\left(2 \mathrm{~s}, 6 \mathrm{H}, 2 \mathrm{CH}_{3}\right), 7.07(\mathrm{~s}, 1 \mathrm{H}, \mathrm{NH}), 7.09(\mathrm{~s}, 1 \mathrm{H}, \mathrm{CH}=\mathrm{N}), 7.30-7.60$ $\left(\mathrm{m}, 9 \mathrm{H}, \mathrm{C}_{6} \mathrm{H}_{5}, \mathrm{C}_{6} \mathrm{H}_{4}\right), 10.01,11.97(2 \mathrm{~s}, 2 \mathrm{H}, 2 \mathrm{NH}) .{ }^{13} \mathrm{C}$ NMR $\left(\mathrm{DMSO}-\mathrm{d}_{6}\right) \delta 19.8,21.6\left(2 \mathrm{CH}_{3}\right)$, $116.4(\mathrm{CN}), 120.4,121.8,122.0,124.1,124.9,130.6,133.9,134.3,138.8,140.1,144.9\left(\mathrm{C}_{6} \mathrm{H}_{5}\right.$, $\mathrm{C}_{6} \mathrm{H}_{4}$, thiophene $\left.\mathrm{C}\right), 164.1,164.8(2 \mathrm{CO}), 172.1(\mathrm{C}=\mathrm{N})$.

5-Acetamido-4-cyano-3-((2-(4-methoxyphenyl) hydrazono) methyl)-N-phenylthiophene-2carboxamide (9c). Yield $3.76 \mathrm{~g}(87 \%)$; m.p. $280^{\circ} \mathrm{C}$; anal. calcd. for $\mathrm{C}_{22} \mathrm{H}_{19} \mathrm{~N}_{5} \mathrm{O}_{3} \mathrm{~S}(433.48)$ : $\mathrm{C}$, $60.96 ; \mathrm{H}, 4.42 ; \mathrm{N}, 16.16$; S, 7.40\%; found: $\mathrm{C}, 60.37 ; \mathrm{H}, 4.36 ; \mathrm{N}, 16.09 ; \mathrm{S}, 7.66 \%$. ${ }^{1} \mathrm{H}$ NMR (DMSO-d $\left.)_{6}\right) \delta 2.50,3.30\left(2 \mathrm{~s}, 6 \mathrm{H}, 2 \mathrm{CH}_{3}\right), 6.93(\mathrm{~s}, 1 \mathrm{H}, \mathrm{CH}), 7.32-7.71\left(\mathrm{~m}, 9 \mathrm{H}, \mathrm{C}_{6} \mathrm{H}_{5}\right.$, $\left.\mathrm{C}_{6} \mathrm{H}_{4}\right), 9.93,9.94,11.95(3 \mathrm{H}, 3 \mathrm{H}, 3 \mathrm{NH}) .{ }^{13} \mathrm{C} \mathrm{NMR}\left(\mathrm{DMSO}_{-} \mathrm{d}_{6}\right) \delta 19.8,22.4\left(2 \mathrm{CH}_{3}\right), 116.8(\mathrm{CN})$, $120.5,121.1,122.3,124.4,124.9,130.6,132.3,133.6,138.9,140.3,144.5\left(\mathrm{C}_{6} \mathrm{H}_{5}, \mathrm{C}_{6} \mathrm{H}_{4}\right.$, thiophene C), 164.3, $164.9(2 \mathrm{CO}), 172.3(\mathrm{C}=\mathrm{N})$.

5-Acetamido-3-((2-(4-chlorophenyl)hydrazono)methyl)-4-cyano- $N$-phenylthiophene-2-carboxamide (9d). Yield $3.63 \mathrm{~g} \mathrm{(83 \% );} \mathrm{m.p.} 295^{\circ} \mathrm{C}$; anal. calcd for $\mathrm{C}_{21} \mathrm{H}_{16} \mathrm{ClN}_{5} \mathrm{O}_{2} \mathrm{~S}$ (437.9): C, 57.60; $\mathrm{H}, 3.68$; N, 15.99; S, 7.32\%; found: C, 57.76; H, 3.45; N, 16.09; S, 7.46\%. ${ }^{1} \mathrm{H}$ NMR (DMSO$\left.\mathrm{d}_{6}\right) \delta 2.25\left(\mathrm{~s}, 3 \mathrm{H}, \mathrm{CH}_{3}\right), 7.07(\mathrm{~s}, 1 \mathrm{H}, \mathrm{NH}), 7.09(\mathrm{~s}, 1 \mathrm{H}, \mathrm{CH}=\mathrm{N}), 7.30-7.60\left(\mathrm{~m}, 9 \mathrm{H}, \mathrm{C}_{6} \mathrm{H}_{5}, \mathrm{C}_{6} \mathrm{H}_{4}\right)$, 10.01, 11.97 (2s, 2H, 2NH). ${ }^{13} \mathrm{C}$ NMR $\left(\mathrm{DMSO}_{6}\right) \delta 21.9\left(\mathrm{CH}_{3}\right), 116.6(\mathrm{CN}), 120.6,121.4$, 122.8, 124.2, 124.5, 130.8, 132.1, 133.6, 138.9, 140.2, $144.9\left(\mathrm{C}_{6} \mathrm{H}_{5}, \mathrm{C}_{6} \mathrm{H}_{4}\right.$, thiophene $\left.\mathrm{C}\right), 164.6$, $164.8(2 \mathrm{CO}), 172.2(\mathrm{C}=\mathrm{N})$.

3,4-Dihydro-4-imino-2-mercapto-5-methyl-N,3-diphenylthieno[2,3-d]pyrimidine-6-carboxamide (12)

To a solution of $3(2.57 \mathrm{~g}, 0.01 \mathrm{~mol})$ in absolute ethanol $(40 \mathrm{~mL})$ containing triethylamine $(1.0$ $\mathrm{mL})$, phenyl isothiocyanate $(1.35 \mathrm{~g}, 0.01 \mathrm{~mol})$ was added. The reaction mixture was heated underreflux for $2 \mathrm{~h}$ then left to cool. The solid product formed upon evaporating the solution under vacuum followed by triturating the remaining product with ethanol, was collected by filtration and dried. The obtained product was crystallized from ethanol to give pale yellow crystals. Yield $2.70 \mathrm{~g}(75 \%)$; m.p. $120-135{ }^{\circ} \mathrm{C}$; anal. calcd. for $\mathrm{C}_{20} \mathrm{H}_{16} \mathrm{~N}_{4} \mathrm{OS}_{2}$ (392.5): C, 61.20; $\mathrm{H}, 4.11$; N, 14.27; S, 16.34\%; found: C, 61.31; H, 4.21; N, 14.38; S, 16.44\%. IR (KBr) v/cm ${ }^{-1}$ 3480-3320 (SH, 2NH), $3055\left(\mathrm{CH}\right.$ aromatic), $1688(\mathrm{CO}) 1660($ exocyclic $\mathrm{C}=\mathrm{N}), 1628(\mathrm{C}=\mathrm{C}) ;{ }^{1} \mathrm{H}$ NMR (DMSO-d d $_{6} \delta 2.51\left(\mathrm{~s}, 3 \mathrm{H}, \mathrm{CH}_{3}\right), 7.30-7.66\left(\mathrm{~m}, 10 \mathrm{H}, 2 \mathrm{C}_{6} \mathrm{H}_{5}\right), 7.71(\mathrm{~s}, 1 \mathrm{H}, \mathrm{SH}), 9.54,11.08$ $(2 \mathrm{~s}, 2 \mathrm{H}, 2 \mathrm{NH}) .{ }^{13} \mathrm{C}$ NMR $\left(\mathrm{DMSO}-\mathrm{d}_{6}\right) \delta 19.6\left(\mathrm{CH}_{3}\right), 119.8,120.3,122.4,123.7,126.0,128.9$, $130.2,131.2,133.8,138.9,140.2,142.6,144.6,146.8$ (two $\mathrm{C}_{6} \mathrm{H}_{5}$, thiophene C), 164.2 (CO), $174.8(\mathrm{C}=\mathrm{N})$.

3-Amino-4,6-dihydro-4-imino- $N$-phenylthieno[3,4-c] thiophene-1-carboxamide (14)

To a solution of $3(2.57 \mathrm{~g}, 0.01 \mathrm{~mol})$ in absolute ethanol $(50 \mathrm{~mL})$ containing triethylamine $(2.0$ $\mathrm{mL})$, element sulfur $(3.2 \mathrm{~g}, 0.1 \mathrm{~mol})$ was added. The reaction mixture was heated under reflux for $3 \mathrm{~h}$ then left to cool. The formed solid product was collected by filtration and dried. The obtained product was crystallized from ethanol to give pale yellow crystals. Yield $2.02 \mathrm{~g}(70 \%)$; m.p. $190{ }^{\circ} \mathrm{C}$; anal. calcd. for $\mathrm{C}_{13} \mathrm{H}_{11} \mathrm{~N}_{3} \mathrm{OS}_{2}$ (289.38): $\mathrm{C}, 53.96 ; \mathrm{H}, 3.83 ; \mathrm{N}, 14.52 ; \mathrm{S}, 22.16 \%$; found: C, 52.77; H, 3.67; N, 14.69; S, 22.09\%. IR (KBr) v/cm- ${ }^{1} 3364-3316\left(\mathrm{NH}_{2}, \mathrm{NH}\right), 3057$ $\left(\mathrm{CH}\right.$, aromatic), $1718(\mathrm{C}=\mathrm{O}), 1624(\mathrm{C}=\mathrm{N}) ;{ }^{1} \mathrm{H}$ NMR $\left(\mathrm{DMSO}-\mathrm{d}_{6}\right) \delta 3.56\left(\mathrm{~s}, 2 \mathrm{H}, \mathrm{NH}_{2}\right), 7.05(\mathrm{~s}$, $1 \mathrm{H}$, thiophene $\left.\mathrm{CH}_{2}\right), 7.27-7.60\left(\mathrm{~m}, 5 \mathrm{H}, \mathrm{C}_{6} \mathrm{H}_{5}\right), 7.71,9.54(2 \mathrm{H}, 2 \mathrm{H}, 2 \mathrm{NH}) .{ }^{13} \mathrm{C}$ NMR (DMSO$\left.\mathrm{d}_{6}\right) \delta 64.6\left(\mathrm{CH}_{2}\right), 119.4,120.8,122.6,124.2,126.5,128.4,131.2,133.8,138.9,140.2,142.6$, 144.6 $\left(\mathrm{C}_{6} \mathrm{H}_{5}\right.$, thiophene $\left.\mathrm{C}\right), 164.8(\mathrm{CO}), 175.9(\mathrm{C}=\mathrm{N})$. 
To a solution of $3(2.57 \mathrm{~g}, 0.01 \mathrm{~mol})$ in absolute ethanol $(50 \mathrm{~mL})$ containing triethylamine $(1.5$ $\mathrm{mL})$, malononitrile $(0.66 \mathrm{~g}, 0.01 \mathrm{~mol})$ was added. The reaction mixture was heated under reflux for $3 \mathrm{~h}$. The solid product formed upon evaporating the solution under vacuum followed by triturating the remaining product with ethanol, was collected by filtration and dried. The obtained product was crystallized from ethanol to give pale brown crystals. Yield $2.45 \mathrm{~g}(76 \%)$; m.p. $185^{\circ} \mathrm{C}$; anal. calcd. for $\mathrm{C}_{16} \mathrm{H}_{13} \mathrm{~N}_{5} \mathrm{OS}$ (323.37): C, 59.43; H, 4.05; N, 21.66; S, 9.92\%; found: C, 59.21; H, 3.89; N, 21.88; S, 10.05\%. IR ( KBr) v/cm $\mathrm{cm}^{-1} 3424-3282\left(2 \mathrm{NH}_{2}, \mathrm{NH}\right), 3056$ $\left(\mathrm{CH}\right.$, aromatic), $2220(\mathrm{CN}), 1680(\mathrm{C}=\mathrm{O}), 1629(\mathrm{C}=\mathrm{C}) .{ }^{1} \mathrm{H}$ NMR $\left(\mathrm{DMSO}-\mathrm{d}_{6}\right) \delta 2.51(\mathrm{~s}, 3 \mathrm{H}$, $\left.\mathrm{CH}_{3}\right), 2.44,3.56\left(2 \mathrm{~s}, 4 \mathrm{H}, \mathrm{NH}_{2}\right), 7.05-7.64\left(\mathrm{~m}, 5 \mathrm{H}, \mathrm{C}_{6} \mathrm{H}_{5}\right), 9.54(\mathrm{~s}, 1 \mathrm{H}, \mathrm{NH}),{ }^{13} \mathrm{C}$ NMR (DMSO$\left.\mathrm{d}_{6}\right) \delta 22.3\left(\mathrm{CH}_{3}\right), 116.9(\mathrm{CN}), 119.4,121.3,122.6,124.2,126.8,129.1,130.5,133.8,138.3$, 140.1, 143.8, $144.1\left(\mathrm{C}_{6} \mathrm{H}_{5}\right.$, thiophene, pyridine $\left.\mathrm{C}\right), 164.2(\mathrm{CO}), 172.2(\mathrm{C}=\mathrm{N})$.

4-Cyano-5-(2-(2,4-dioxopentan-3-ylidene) hydrazinyl)-3-methyl-N-phenylthiophene-2-carboxamide (20)

To a cold solution of acetyl acetone $(1.00 \mathrm{~g}, 0.01 \mathrm{~mol})$ in ethanol $(40 \mathrm{~mL})$ containing sodium hydroxide $(2.5 \mathrm{~g})$ a cold solution of the respective diazonium salt [prepared by the addition of sodium nitrite solution $(0.70 \mathrm{~g}, 0.01 \mathrm{~mol})$ to a cold solution of $\mathbf{3}(2.57 \mathrm{~g}, 0.01 \mathrm{~mol})$ in concentrated hydrochloric acid $(12 \mathrm{~mL})$ with continuous stirring] was added while stirring. The formed solid product, upon stirring at room temperature for $1 \mathrm{~h}$ was collected by filtration and dried. The obtained product was crystallized from ethanol to give black crystals. Yield $2.87 \mathrm{~g}$ (78\%); m.p. $160-170{ }^{\circ} \mathrm{C}$; anal. calcd. for $\mathrm{C}_{18} \mathrm{H}_{16} \mathrm{~N}_{4} \mathrm{O}_{3} \mathrm{~S}$ (368.41): C, 58.68; H, 4.38; N, 15.21; S, 8.70\%; found: C, 58.89; H, 4.25; N, 15.53; S, 8.88\%. IR (KBr) v/cm ${ }^{-1} 3422-3280(2 \mathrm{NH}), 3053$ $\left(\mathrm{CH}\right.$, aromatic), $2222(\mathrm{CN}), 1684(\mathrm{C}=\mathrm{O}), 1622(\mathrm{C}=\mathrm{C}) ;{ }^{1} \mathrm{H}$ NMR $\left(\mathrm{DMSO}-\mathrm{d}_{6}\right) \delta 2.32,2.45,2.62$ $\left(3 \mathrm{~s}, 9 \mathrm{H}, 3 \mathrm{CH}_{3}\right), 7.27-7.62\left(\mathrm{~m}, 5 \mathrm{H}, \mathrm{C}_{6} \mathrm{H}_{5}\right), 7.72,9.54(2 \mathrm{~s}, 2 \mathrm{H}, 2 \mathrm{NH}),{ }^{13} \mathrm{C}$ NMR $\left(\mathrm{DMSO}-\mathrm{d}_{6}\right)$ $\delta 19.8,20.6,21.3\left(3 \mathrm{CH}_{3}\right), 117.0(\mathrm{CN}), 120.4,121.7,122.9,124.7,125.9,128.2,130.1,138.0$ $\left(\mathrm{C}_{6} \mathrm{H}_{5}\right.$, thiophene $\left.\mathrm{C}\right), 163.2,164.0,165.2(3 \mathrm{CO}), 173.2(\mathrm{C}=\mathrm{N})$.

4-Cyano-5-(2-cyanoacetamido)-3-methyl-N-phenylthiophene-2-carboxamide (22)

To a solution of $3(2.57 \mathrm{~g}, 0.01 \mathrm{~mol})$ in absolute ethanol $(40 \mathrm{~mL})$ containing triethylamine $(1.5$ $\mathrm{mL})$, ethyl cyanoacetate $(1.13 \mathrm{~g}, 0.01 \mathrm{~mol})$ was added. The reaction mixture was heated under reflux for $2 \mathrm{~h}$. The solid product formed upon pouring onto ice/water containing a few drops of hydrochloric acid was collected by filtration and dried. The obtained product was crystallized from ethanol to give brown crystals. Yield $2.59 \mathrm{~g}(80 \%)$; m.p. $195{ }^{\circ} \mathrm{C}$; anal. calcd. for $\mathrm{C}_{16} \mathrm{H}_{12} \mathrm{~N}_{4} \mathrm{O}_{2} \mathrm{~S}$ (324.36): C, 59.25; H, 3.73; N, 17.27; S, 9.89\%; found: $\mathrm{C}, 59.86 ; \mathrm{H}, 3.65 ; \mathrm{N}$, 17.06 ; S, 9.93. IR (KBr) v/cm ${ }^{-1} 3477-3318(2 \mathrm{NH}), 3045$ (CH aromatic); ${ }^{1} \mathrm{H}$ NMR (DMSO-d $\left.\mathrm{d}_{6}\right) \delta$ $2.54\left(\mathrm{~s}, 3 \mathrm{H}, \mathrm{CH}_{3}\right), 4.42\left(\mathrm{~s}, 2 \mathrm{H}, \mathrm{CH}_{2}\right) .7 .09-7.60\left(\mathrm{~m}, 5 \mathrm{H}, \mathrm{C}_{6} \mathrm{H}_{5}\right), 7.71,9.54(2 \mathrm{~s}, 2 \mathrm{H}, 2 \mathrm{NH}) .{ }^{13} \mathrm{C}$ NMR $(\text { DMSO-d })_{6} \delta 20.6\left(\mathrm{CH}_{3}\right), 62.1\left(\mathrm{CH}_{2}\right), 116.8,117.3(2 \mathrm{CN}), 119.9,120.6,121.9,123.1$, $124.7,125.5,126.8,130.2,138.3,140.2\left(\mathrm{C}_{6} \mathrm{H}_{5}\right.$, thiophene $\left.\mathrm{C}\right), 164.4,164.5(2 \mathrm{CO})$.

5-(2-Cyano-3-phenylacrylamido)-4-cyano-3-methyl-N-phenylthiophene-2-carboxamide (23)

To a solution of $22(3.24 \mathrm{~g}, 0.01 \mathrm{~mol})$ in 1,4-dioxane $(40 \mathrm{~mL})$ containing piperidine $(1.0 \mathrm{~mL})$, benzaldehyde $(1.0 \mathrm{~g}, 0.01 \mathrm{~mol})$ was added. The reaction mixture was heated under reflux for $3 \mathrm{~h}$ then left to cool. The solid product formed upon evaporating the solution under vacuum followed by triturating the remaining product with ethanol, was collected by filtration and dried. The obtained product was crystallized from 1,4-dioxane to give yellowish green crystals. Yield $3.37 \mathrm{~g}(82 \%)$; m.p. $175^{\circ} \mathrm{C}$; anal. calcd. for $\mathrm{C}_{23} \mathrm{H}_{16} \mathrm{~N}_{4} \mathrm{O}_{2} \mathrm{~S}$ (412.46): C, 66.97; H, 3.91; N, 13.58; 
Uses of acetoacetanilide for the synthesis of thiophene derivatives with cytotoxic activities 531

S, 7.77\%; found: C, 66.87; H, 3.69; N, 13.73; S, 8.01\%. IR (KBr) v/cm $\mathrm{cm}^{-1} 3424-3362(2 \mathrm{NH})$, $3059\left(\mathrm{CH}\right.$, aromatic), $2924\left(\mathrm{CH}_{3}\right), 1687,1685(2 \mathrm{C}=\mathrm{O}), 1657(\mathrm{C}=\mathrm{C}) ;{ }^{1} \mathrm{H}$ NMR (DMSO$\left.\mathrm{d}_{6}\right) \delta 2.53\left(\mathrm{~s}, 3 \mathrm{H}, \mathrm{CH}_{3}\right), 7.11(\mathrm{~s}, 1 \mathrm{H}, \mathrm{CH}=\mathrm{C}), 7.39-7.68\left(\mathrm{~m}, 10 \mathrm{H}, 2 \mathrm{C}_{6} \mathrm{H}_{5}\right), 8.88,10.27(2 \mathrm{~s}, 2 \mathrm{H}$, $2 \mathrm{NH}) .{ }^{13} \mathrm{C}$ NMR (DMSO-d $\left.{ }_{6}\right) \delta 19.6\left(\mathrm{CH}_{3}\right), 87.3,92.5(\mathrm{CH}=\mathrm{C}), 116.4,116.9(2 \mathrm{CN}), 119.3$, $120.8,122.4,123.6,124.8,125.9,126.8,130.1,132.1,136.2,140.9,142.6$ (two $\mathrm{C}_{6} \mathrm{H}_{5}$, thiophene C), 164.6, $166.2(2 \mathrm{CO})$.

N-(3-Cyano-4-methyl-5-(phenylcarbamoyl)thiophen-2-yl)-2-oxo-2H-chromene-3-carboxamide (25)

To a solution of $22(3.24 \mathrm{~g}, 0.01 \mathrm{~mol})$ in 1,4-dioxane $(40 \mathrm{~mL})$ containing piperidine $(1.0 \mathrm{~mL})$, salicyaldehyde $(1.22 \mathrm{~g}, 0.01 \mathrm{~mol})$ was added. The reaction mixture was heated under reflux for $2 \mathrm{~h}$ then left to cool. The solid product formed upon evaporating the solution under vacuum followed by triturating the remaining product with ethanol, was collected by filtration and dried. The obtained product was crystallized from ethanol to give orange yellow crystals. Yield $3.00 \mathrm{~g}$ (70\%); m.p. 240-250 ${ }^{\circ} \mathrm{C}$; anal. calcd. for $\mathrm{C}_{23} \mathrm{H}_{15} \mathrm{~N}_{3} \mathrm{O}_{4} \mathrm{~S}$ (429.45): C, 64.33; H, 3.52; N, 9.78; S, 7.47\%; found: C, 64.27; H, 3.34; N, 9.92; S, 7.64\%. IR (KBr) v/cm ${ }^{-1} 3479-3321$ (2NH), 3053 $\left(\mathrm{CH}\right.$, aromatic), $2921\left(\mathrm{CH}_{3}\right), 2220(\mathrm{CN}), 1689-1685(3 \mathrm{C}=\mathrm{O}), 1653(\mathrm{C}=\mathrm{C}) .{ }^{1} \mathrm{H}$ NMR (DMSO$\left.\mathrm{d}_{6}\right) \delta 2.53\left(\mathrm{~s}, 3 \mathrm{H}, \mathrm{CH}_{3}\right), 7.00(\mathrm{~s}, \mathrm{H}$, coumarin $\mathrm{H}-4), 7.34-7.90\left(\mathrm{~m}, 9 \mathrm{H}, \mathrm{C}_{6} \mathrm{H}_{5}, \mathrm{C}_{6} \mathrm{H}_{4}\right), 9.07,10.24$ $(2 \mathrm{~s}, 2 \mathrm{H}, 2 \mathrm{NH}) .{ }^{13} \mathrm{C}$ NMR $\left(\mathrm{DMSO}_{-} \mathrm{d}_{6}\right) \delta 18.3\left(\mathrm{CH}_{3}\right), 116.3(\mathrm{CN}), 96.8$ (coumarin C-4), 120.5, $121.2,121.9,123.9,125.2,125.6,129.1,130.4,132.7,134.9,139.2,140.9,141.8,143.6,152.0$ $\left(\mathrm{C}_{6} \mathrm{H}_{5}\right.$, coumarin, thiophene $\left.\mathrm{C}\right), 164.1,165.9,166.3$ (3CO).

General procedure for the synthesis of 2-cyanoacetamido)-4-cyano-3-methyl- $N$ phenylthiophene-2-carboxamide derivatives $\mathbf{2 6 a - d}$

To a cold solution of $22(3.24 \mathrm{~g}, 0.01 \mathrm{~mol})$ in 1,4-dioxane $(40 \mathrm{~mL})$ containing sodium hydroxide $(2.5 \mathrm{~g})$ a cold solution of the respective diazonium salt [prepared by the addition of sodium nitrite solution $(0.70 \mathrm{~g}, 0.01 \mathrm{~mol})$ to a cold solution of either aniline $(0.94 \mathrm{~g}, 0.01 \mathrm{~mol}), p$ toluidine (1.15 g, $0.01 \mathrm{~mol})$, $p$-methoxy aniline $(1.3 \mathrm{~g}, 0.01 \mathrm{~mol})$ or $p$-chloroaniline $(1.29 \mathrm{~g}, 0.01$ $\mathrm{mol})$ in concentrated hydrochloric acid $(12 \mathrm{~mL})$ with continuous stirring]was added while stirring. The formed solid product, in each case, upon stirring at room temperature for $1 \mathrm{~h}$ was collected by filtration and dried. The obtained product was crystallized from ethanol to give brown crystals for 26a and 26c and black crystals for $\mathbf{2 6 b}$ and $\mathbf{2 6 d}$.

2-(2-Phenylhydrazono)-2-cyanoacetamido)-4-cyano-3-methyl-N-phenylthiophene-2-carboxamide (26a). Yield $3.5 \mathrm{~g}(82 \%)$; m.p. $70-80{ }^{\circ} \mathrm{C}$; anal. calcd. for $\mathrm{C}_{22} \mathrm{H}_{16} \mathrm{~N}_{6} \mathrm{O}_{2} \mathrm{~S}$ (428.47): C, 61.67; H, 3.76; N, 19.61; S, 7.48. found: C, 61.21; H, 3.58; N, 19.88; S, 7.58\%. IR (KBr) v/cm $\mathrm{cm}^{-1} 3492$ $3329(3 \mathrm{NH}), 3056(\mathrm{CH}$, aromatic), $2923(\mathrm{CH} 3), 2222,2220(2 \mathrm{CN}), 1689,1687(2 \mathrm{C}=\mathrm{O}), 1660$ $(\mathrm{C}=\mathrm{N}), 1633(\mathrm{C}=\mathrm{C}) .{ }^{1} \mathrm{H}$ NMR $\left(\mathrm{DMSO}_{-} \mathrm{d}_{6}\right) \delta 2.52\left(\mathrm{~s}, 3 \mathrm{H}, \mathrm{CH}_{3}\right), 7.30(\mathrm{~s}, 1 \mathrm{H}, \mathrm{NH}), 7.40-7.66(\mathrm{~m}$, $\left.10 \mathrm{H}, 2 \mathrm{C}_{6} \mathrm{H}_{5}\right), 8.40,9.48(2 \mathrm{~s}, 2 \mathrm{H}, 2 \mathrm{NH}) .{ }^{13} \mathrm{C} \mathrm{NMR}\left(\mathrm{DMSO}_{6}\right) \delta 18.8(\mathrm{CH} 3), 116.7,117.2$ $(2 \mathrm{CN}), 121.0,121.8,122.3,123.5,124.2,125.9,129.1,132.7,132.9,140.9,141.8,143.6$ (two C6H5, thiophene $\mathrm{C}), 164.4,165.2(2 \mathrm{CO}), 172.0(\mathrm{C}=\mathrm{N})$.

2-(2-p-Tolylhydrazono)-2-cyanoacetamido)-4-cyano-3-methyl-N-phenylthiophene-2-carbox-

amide (26b). Yield $3.53 \mathrm{~g} \mathrm{(80 \% );} \mathrm{m.p.} 110-120^{\circ} \mathrm{C}$; anal. calcd. for $\mathrm{C}_{23} \mathrm{H}_{18} \mathrm{~N}_{6} \mathrm{O}_{2} \mathrm{~S}$ (442.49): $\mathrm{C}$, 62.43; H, 4.10; N, 18.99; S, 7.25\%; found: C, 62.38; H, 3.91; N, 19.06; S, 7.41\%. IR (KBr) $\mathrm{v} / \mathrm{cm}^{-1} 3488-3339(3 \mathrm{NH}), 3053\left(\mathrm{CH}\right.$, aromatic), $2923\left(\mathrm{CH}_{3}\right), 2222,2220(2 \mathrm{CN}), 1693,1689$ $(2 \mathrm{C}=\mathrm{O}), 1661(\mathrm{C}=\mathrm{N}), 1630(\mathrm{C}=\mathrm{C}) .{ }^{1} \mathrm{H}$ NMR $\left(\mathrm{DMSO}_{-} \mathrm{d}_{6}\right) \delta 2.39,2.40\left(2 \mathrm{~s}, 6 \mathrm{H}, 2 \mathrm{CH}_{3}\right), 7.09(\mathrm{~s}$, $1 \mathrm{H}, \mathrm{NH}), 7.37-7.71\left(\mathrm{~m}, 9 \mathrm{H}, \mathrm{C}_{6} \mathrm{H}_{5}, \mathrm{C}_{6} \mathrm{H}_{4}\right), 8.80,10.13(2 \mathrm{~s}, 2 \mathrm{H}, 2 \mathrm{NH}) .{ }^{13} \mathrm{C}$ NMR $\left(\mathrm{DMSO}_{6}\right) \delta$ 
$18.3,19.6\left(2 \mathrm{CH}_{3}\right), 116.2,117.0(2 \mathrm{CN}), 119.8,122.0,122.3,123.8,124.6,126.4,129.8,132.2$, 133.6, 140.5, 141.8, 143.8 (two $\mathrm{C}_{6} \mathrm{H}_{5}$, thiophene $\left.\mathrm{C}\right), 164.2,165.7(2 \mathrm{CO}), 172.1(\mathrm{C}=\mathrm{N})$.

2-(2-(4-Methoxyphenyl)hydrazono)-2-cyanoacetamido)-4-cyano-3-methyl-N-phenylthiophene-2carboxamide (26c). Yield 3.89 g (85\%); m.p. $155^{\circ} \mathrm{C}$; anal. calcd. for $\mathrm{C}_{23} \mathrm{H}_{18} \mathrm{~N}_{6} \mathrm{O}_{3} \mathrm{~S}(458.49)$ : $\mathrm{C}$, $60.25 ; \mathrm{H}, 3.96 ; \mathrm{N}, 18.33$; S, 6.99\%; found: C, 59.89; H, 3.78; N, 18.09; S, 7.11\%. IR (KBr) v/cm ${ }^{-1} 3447-3267(3 \mathrm{NH}), 3052\left(\mathrm{CH}\right.$, aromatic), $2970\left(\mathrm{CH}_{3}\right), 2223(\mathrm{CN}), 1704(\mathrm{C}=\mathrm{O}), 1643$ $(\mathrm{C}=\mathrm{N}), 1529(\mathrm{C}=\mathrm{C}) .{ }^{1} \mathrm{H}$ NMR $\left(\mathrm{DMSO}_{6}\right) \delta 2.49,2.68\left(2 \mathrm{~s}, 6 \mathrm{H}, 2 \mathrm{CH}_{3}\right), 7.09(\mathrm{~s}, 1 \mathrm{H}, \mathrm{NH}), 7.39-$ $7.79\left(\mathrm{~m}, 9 \mathrm{H}, \mathrm{C}_{6} \mathrm{H}_{5}, \mathrm{C}_{6} \mathrm{H}_{4}\right), 10.55,11.11(2 \mathrm{~s}, 2 \mathrm{H}, 2 \mathrm{NH}) .{ }^{13} \mathrm{C}$ NMR (DMSO-d $\left.{ }_{6}\right) \delta 18.6,21.3$ $\left(2 \mathrm{CH}_{3}\right), 116.4,117.3(2 \mathrm{CN}), 120.9,122.9,122.3,123.8,124.8,126.4,129.3,132.4,133.8$, $140.9,141.3,143.1$ (two $\mathrm{C}_{6} \mathrm{H}_{5}$, thiophene $\left.\mathrm{C}\right), 164.3,165.9(2 \mathrm{CO}), 172.0(\mathrm{C}=\mathrm{N})$.

5-(2-(2-(4-Chlorophenyl)hydrazono)-2-cyanoacetamido)-4-cyano-3-methyl-N-phenylthiophene2-carboxamide (26d). Yield $3.65 \mathrm{~g}(79 \%)$; m.p. $135^{\circ} \mathrm{C}$; anal. calcd. for $\mathrm{C}_{22} \mathrm{H}_{15} \mathrm{ClN}_{6} \mathrm{O}_{2} \mathrm{~S}(462.91)$ : C, 57.08; H, 3.27; N, 18.15; S, 6.93\%; found: C, 56.82; H, 3.12; N, 17.99; S, 7.14\%. IR (KBr) $v / \mathrm{cm}^{-1} 3476-3320(3 \mathrm{NH}), 3055\left(\mathrm{CH}\right.$, aromatic), $2924\left(\mathrm{CH}_{3}\right), 2224,2220(2 \mathrm{CN}), 1690,1689$ $(2 \mathrm{C}=\mathrm{O}), 1661(\mathrm{C}=\mathrm{N}), 1624(\mathrm{C}=\mathrm{C}) .{ }^{1} \mathrm{H}$ NMR $\left(\mathrm{DMSO}_{-} \mathrm{d}_{6}\right) \delta 2.62\left(\mathrm{~s}, 3 \mathrm{H}, \mathrm{CH}_{3}\right), 7.06(\mathrm{~s}, 1 \mathrm{H}, \mathrm{NH})$, 7.30-7.79 (m, 9H, $\left.\mathrm{C}_{6} \mathrm{H}_{5}, \mathrm{C}_{6} \mathrm{H}_{4}\right), 10.27,11.10(2 \mathrm{~s}, 2 \mathrm{H}, 2 \mathrm{NH}) .{ }^{13} \mathrm{C}$ NMR (DMSO-d $\left.\mathrm{d}_{6}\right) \delta 18.9$ $\left(\mathrm{CH}_{3}\right), 116.2$, 117.1 (2CN), 120.8, 122.5, 122.8, 123.1, 124.8, 126.2, 129.3, 132.6, 133.8, 140.3, 142.8, 143.7 (two $\mathrm{C}_{6} \mathrm{H}_{5}$, thiophene $\mathrm{C}$ ), 164.6, $165.4(2 \mathrm{CO}), 172.1(\mathrm{C}=\mathrm{N})$.

3,5-Diamino-4-cyano-N-(3-cyano-4-methyl-5-(phenylcarbamoyl)thiophen-2-yl)thiophene-2carboxamide (27)

To a solution of $22(3.24 \mathrm{~g}, 0.01 \mathrm{~mol})$ in 1,4-dioxane $(50 \mathrm{~mL})$ containing triethylamine $(1.5$ $\mathrm{mL})$, malononitrile $(0.66 \mathrm{~g}, 0.01 \mathrm{~mol})$ was added followed by elemental sulfur $(0.32 \mathrm{~g}, 0.01$ $\mathrm{mol}$ ). The reaction mixture was heated under reflux for $3 \mathrm{~h}$. The solid product formed upon evaporating the solution under vacuum followed by triturating the remaining product with ethanol, was collected by filtration and dried. The obtained product was crystallized from ethanol to give pale brown crystals. Yield $3.16 \mathrm{~g}(75 \%)$; m.p. $185{ }^{\circ} \mathrm{C}$; anal. calcd. for $\mathrm{C}_{19} \mathrm{H}_{14} \mathrm{~N}_{6} \mathrm{O}_{2} \mathrm{~S}_{2}$ (422.48): C, 54.01; H, 3.34; N, 19.89; S, 15.18\%; found: C, 53.72; H, 3.28; N, 20.11; S, 15.23. IR (KBr) $v / \mathrm{cm}^{-1} 3491-3316(3 \mathrm{NH}), 3056\left(\mathrm{CH}\right.$, aromatic), $2921\left(\mathrm{CH}_{3}\right), 2227$, $2220(2 \mathrm{CN}), 1692,1686(2 \mathrm{C}=\mathrm{O}), 1660(\mathrm{C}=\mathrm{N}), 1623(\mathrm{C}=\mathrm{C}) .{ }^{1} \mathrm{H}$ NMR $\left(\mathrm{DMSO}-\mathrm{d}_{6}\right) \delta 2.50(\mathrm{~s}, 3 \mathrm{H}$, $\left.\mathrm{CH}_{3}\right), 3.45-3.48\left(2 \mathrm{~s}, 4 \mathrm{H}, 2 \mathrm{NH}_{2}\right) .7 .03-7.60\left(\mathrm{~m}, 5 \mathrm{H}, \mathrm{C}_{6} \mathrm{H}_{5}\right), 7.70,9.54(2 \mathrm{~s}, 2 \mathrm{H}, 2 \mathrm{NH}) .{ }^{13} \mathrm{C} \mathrm{NMR}$ $\left(\mathrm{DMSO}_{-} \mathrm{d}_{6}\right) \delta 18.6\left(\mathrm{CH}_{3}\right), 116.1,116.9(2 \mathrm{CN}), 120.9,122.8,123.0,124.3,124.8,126.8,129.3$, $132.9,136.2,140.7,142.3,143.1\left(\mathrm{C}_{6} \mathrm{H}_{5}\right.$, two thiophene $\left.\mathrm{C}\right), 164.3,165.7$ (2CO).

\section{CONCLUSION}

A series of thiophene derivatives were synthesized and evaluated against three cancer cell lines. The above results allow the conclusion that administration of the tested compounds to the cancer cell line showed promising anticancer activity. Compounds 5a, 5b, 8, 22, and 23 exhibited optimal cytotoxic effect against cancer cell lines.

\section{REFERANCES}

1. Mohanakrishnan, A.K.; Amaladass, P.; Clement, J.A. Synthesis of end-blocked thienyl oligomers incorporating benzo[c]thiophene. Tetrahedron Lett. 2007, 48, 779-784.

2. Ferreira, I.C.; Queiroz M.J., Kirsch, G. Tandem palladium-catalyzed borylation and suzuki coupling (BSC) to thienocarbazole precursors. Tetrahedron Lett. 2003, 44, 4327-4343. 
3. Queiroz, M.J.; Ferreir, I.C.; Gaetano, Y.D.; Kirsch, G.; Calhelha, R.C.; Estevinho, L.M. Synthesis and antimicrobial activity studies of ortho-chlorodiarylamines and heteroaromatic tetracyclic systems in the benzo[b]thiophene series. J. Bioorg. Med. Chem. 2006, 14, 68276848.

4. Sall, D.J.; Briggs, S.L.; Chirgadze, N.Y.; Clawson, D.K.; Gifford-Moore, D.S.; Klimkowski, V.J., McCowan J.R., Smith G.F., Wikel J.H. Dibasic benzo[b]thiophene derivatives as a novel class of active site directed thrombin inhibitors. II: Exploring interactions at the proximal (S2) binding site. Bioorg. Med. Chem. Lett. 1998, 8, 2527-2538.

5. Lee, S.; Lee, H.; Yi, K.Y.; Lee, B.H.; Cho, N.S. 4-Substituted(benzo[b]thiophene-2carbonyl)guanidines as novel $\mathrm{Na}^{+} / \mathrm{H}^{+}$exchanger isoform-1 (NHE-1) inhibitors. Bioorg. Med. Chem. Lett. 2005, 15, 2998-3001.

6. Bastian, J.A.; Chrigadze; N.; Denny, M.L.; Gifford-Moore, D.S.; Sall, D.J.; Smith, G.F.; Wikel, J.H. Diamino benzo[b]thiophene derivatives as a novel class of active site directed thrombin inhibitors. III: Enhancing activity by imposing conformational restriction in the C4" side chain. Bioorg. Med. Chem. Lett. 1999, 9, 363-368.

7. Wang, Y.; Burton, D.J. Site-specific preparation of 4-substituted-6-fluoro(carboalkoxyl) benzo[b]furans and benzo[b]thiophenes via base-catalyzed cyclization of enyne derivatives. J. Fluorine Chem. 2007, 128, 1052-1057.

8. Pinto, E.; Queiroz, M.J.; Vale-Silva, L.A.; Oliveira, J.F.; Begouin, A.; Begouin, J.M.; Kirsch, G. Antifungal activity of synthetic di(hetero)arylamines based on the benzo[b]thiophene moiety. J. Bioorg. Med. Chem. 2008, 16, 8172-8177.

9. Eicher, T.; Hauptman, S.; Speicher, A. The Chemistry of Heterocycles: Chapter 5, Five Membered Heterocycles, Section 5.6 Thiophene, Wiley-VCH: New York; 2003.

10. King, W.J.; Nord, F.F. Studies in the thiophene series. V: Wolff-Kishner reductions. J. Org. Chem. 1949, 14, 638-642.

11. Wu, C.; Decker, E.R.; Blok, N.; Bui, H., You, T.J.; Wang, J.; Bourgoyne, A.R.; Knowles, V.; Berens, K.L.; Holland, G.W.; Brock, T.A.; Dixon, R.A.F. Discovery, modeling, and human pharmacokinetics of $\mathrm{N}$-(2-acetyl-4,6-dimethylphenyl)-3-(3,4-dimethylisoxazol-5ylsulfamoyl)thiophene-2-carboxamide (TBC3711), a second generation, ET A selective, and orally bioavailable endothelin antagonist 1. J. Med. Chem. 2004, 47, 1969-1986.

12. Dure, K.; Dubus, S.; Ho, H.A.; Levesque, I.; Brunette, M.; Corbeil, G.; Boissinot, M.; Boivin, G.; Bergeron, M.G.; Boudreau, D.; Leclerc, M. Fluorescent polymeric transducerfor the rapid, simple, and specific detection of nucleic acids at the zeptomole level. J. Am. Chem. Soc. 2004, 126, 4240-4244.

13. Mohareb, R.M.; Wardakhan, W.W.; Ibrahim, R.A. Synthesis of pyridine, pyran and thiazole containing thiophene derivatives and their anti-tumor evaluations. Med. Chem. Res. 2016, 25, 2187-2204.

14. Mohareb, R.M.; Ibrahim, R.A. Design, cytotoxicity and toxicity of new thiophene and thieno [2,3-b] pyridine derivatives. Med. Chem. Res. 2017, 26, 587-602.

15. Mohareb, R.M.; Abbas N.S., Ibrahim, R.A. New approaches for the synthesis of thiophene derivatives with anti-tumor activities. Acta Chim. Slov. 2013, 60, 583-594.

16. Rost, C.; Karg, S.; Riess, W.; Loi, M.A.; Murgia, M.; Muccini, M. Ambipolar light emitting organic field-effect transistor. Appl. Phys. Lett. 2004, 85, 1613-1615.

17. Vriezema, D.M.; Hoogboom, J.; Veloonia, K.; Takazawa, K.; Christianen, P.C.M.; Maan, J.C.; Rowan, A.E.; Nolte R.J.M. Vesicles and polymerized vesicles from thiophenecontaining rod-coil block copolymers. Angew. Chem. Int. Ed. 2003, 42, 772-776.

18. Yu, H.; Pullen A.E.; Beuschel, M.G.; Swager, T.M. Charge-specific interactions in segmented conducting polymers: An approach to selective ionoresistive responses. Angew. Chem. Int. Ed. 2004, 43, 3700-3703.

19. Sally, E.; Wenzel, M.D. New approaches to anti-inflammatory therapy for asthma. Am. J. Med. 1998, 104, 287-300. 
20. Angerer, E.; Erber, S. 3-Alkyl-2-phenylbenzo[b]thiophenes: Nonsteroidal estrogen antagonists with mammary tumor inhibiting activity. J. Steroid Biochem. Mol. Biol. 1992, 41, 557-562.

21. Malaisa, O.; Neuprez, A.; Reginster, J.Y. Raitements non hormonaux de l'ostéoporose postménopausique. Gynécologie Obstétrique \& Fertilité 2008, 36, 815-822.

22. Luo, Z.; Liu, Z.; Yang, Z. The synthesis and photoactivated cytotoxicity of novel 5-phenyl 3-(2,2':5',2"-terthien-5-yl)-4,5-dihydro-1H-pyrazolines. Chin. Chem. Lett. 2014, 25, 333336.

23. Shchekotikhin, A.E.; Glazunova, V.A.; Dezhenkova, L.G; Luzikov, Y.N.; Sinkevich, Y.B.; Kovalenko, L.V.; Buyanov, V.N.; Balzarini, J.; Huang, F.; Lin, J.; Huang, H.; Shtil, A.A.; Preobrazhenskaya M.N. Synthesis and cytotoxic properties of 4,11-bis[(aminoethyl)amino]anthra[2,3-b]thiophene-5,10-diones, novel analogues of antitumor anthracene-9,10. Bioorg. Med. Chem. 2009, 17, 1861-1869. 